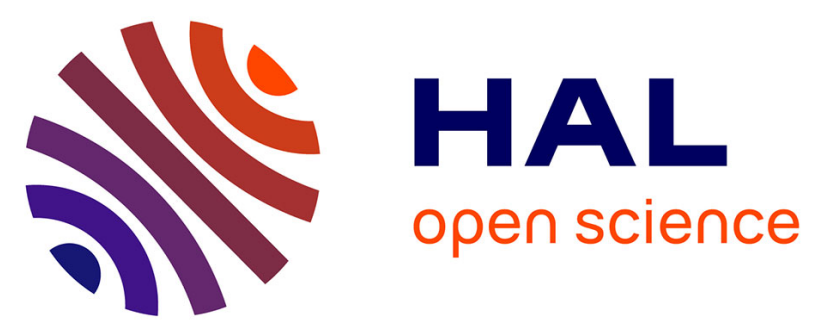

\title{
Hybrid composed method associating conformal transformation with matrix formulation for computing eigenvalues and eigenvectors in bended optical waveguides
}

Lucas Garnier, C Saavedra, Rigoberto Castro -Beltran, José Luis Lucio, Etienne Gaviot, Bruno Bêche

\section{To cite this version:}

Lucas Garnier, C Saavedra, Rigoberto Castro -Beltran, José Luis Lucio, Etienne Gaviot, et al.. Hybrid composed method associating conformal transformation with matrix formulation for computing eigenvalues and eigenvectors in bended optical waveguides. Optik, 2017, 142, pp.536-540. 10.1016/j.ijleo.2017.06.035 . hal-01538177

\section{HAL Id: hal-01538177 \\ https://hal.science/hal-01538177}

Submitted on 15 Jun 2017

HAL is a multi-disciplinary open access archive for the deposit and dissemination of scientific research documents, whether they are published or not. The documents may come from teaching and research institutions in France or abroad, or from public or private research centers.
L'archive ouverte pluridisciplinaire HAL, est destinée au dépôt et à la diffusion de documents scientifiques de niveau recherche, publiés ou non, émanant des établissements d'enseignement et de recherche français ou étrangers, des laboratoires publics ou privés. 


\title{
Hybrid composed method associating conformal transformation with matrix formulation for computing eigenvalues and eigenvectors in bended optical waveguides
}

\author{
L. Garnier ${ }^{\mathrm{a}}$, C. Saavedra ${ }^{\mathrm{b}}$, R. Castro-Beltrán ${ }^{\mathrm{b}}$, J.L. Lucio M. ${ }^{\mathrm{b}}$, E. Gaviot ${ }^{\mathrm{c}}$, B. Bêche ${ }^{\mathrm{a}}$ \\ ${ }^{a}$ Institut de Physique de Rennes - IPR UMR CNRS 6251, Université de Rennes 1, 35042 Rennes, France. \\ ${ }^{\mathrm{b}}$ Universidad de Guanajuato, División de Ciencias e Ingenierías-León, Guanajuato, México. \\ ${ }^{\mathrm{c}}$ Laboratoire d'Acoustique de l'Université du Maine - LAUM UMR CNRS 6613, Université du Maine, 72000 \\ Le Mans, France. \\ E-mails: lucas.garnier@univ-rennes1.fr, bruno.beche@univ-rennes1.fr
}

\begin{abstract}
A fast hybrid composed and numerical method for the calculation of eigenvalues (effective indices) and eigenvectors (optical modes) of a slab waveguide presenting a radius of curvature is presented in this letter. This compound method combines a conformal transformation of the complex plane plus a multilayer matrix formalism addressing directly the modified index profile obtained by the conformal transformation; the matrix formalism is then applied in second step so as to discretize and slice the profile in a virtual multilayer structure. This method being conveniently operable on a personal computer in a short amount of time and is easy to implement. Its results are then compared to values afforded with the commercial vectorial software COMSOL 3D so as to discuss their accuracy.
\end{abstract}

Keywords: Integrated optics, hybrid composed method, mathematic conformal transformation with multilayer formalism methods.

Accurate knowledge of how light behave within a waveguide with a given radius of curvature becomes more and more important because of the development of integrated photonics [1-3], which include ring micro-resonators, phasars, and other devices fitted with a radius of curvature. Sometimes, it can be original and interesting to modify, adapt, enhance or merge various methods of resolution [4,5]. This work presents a hybrid numerical calculation method (HNM) to determine the eigenvalues (effective indices) and eigenvectors related to the optical field within a curved waveguide. This method is a hybrid method which uses at first an apt conformal transformation (CT) of the complex plane plus secondly a matrix multilayer formalism (MMF) developed at the origin for real materials. As a ' $o$ ' composition law, we can write HNM $\equiv($ MMF $o$ CT). The aim of the conformal transformation is to convert a curved geometry into a virtual straight geometry structure; such a conversion entails a significant effect on the index profile of the structure and modifies its pattern. Various kinds of mathematic conformal transformations can be considered [6], and used in physics, optoelectronics and hyper-frequency such as: inversion, rotation, homographic or more particular transformations like the Schwarz-Christoffel or Joukovsky ones; the specific one used in this work will be detailed later. In our case, it will be a shortcut and a mathematical trick that makes it possible to dispense with the 'true' Jacobian method and the equation of propagation in polar coordinates. Then, as regards the modified index profile, we use the matrix multilayer formalism first developed by 
P. Yeh and A. Yariv to describe the behavior of light in stratified structures composed with layers with different indices [7,1]. So as to take advantage from this formalism, the modified index profile obtained thanks to the CT is discretized into virtual layers prior to apply the MMF. In our case, the MMF will be used to follow step by step (discretization) the profile created by the CT. This method allows to make the calculation on a personal computer in a very short amount of time, and its results can be compared to that of the commercial software COMSOL-3D [8].

Let us consider a slab waveguide of width L (Fig. 1) on which will be applied, firstly a conformal transformation (CT) of the complex plane (Fig. 2a) and secondly a matrix multilayer formalism (MMF, Fig. 2b). By way of the conformal transformation, one can go from a $z=x+i y=\rho e^{i \theta}$ complex plane to a pattern transformed as: $w=u+i v=f(z)$. The goal of this transformation is to take advantage of calculations for a non-bended effective waveguide. To achieve this goal, the apt transformation is $\mathrm{w}=\mathrm{R}_{\text {ext }} \ln \left(\mathrm{z} / \mathrm{R}_{\text {ext }}\right)$ with $\mathrm{R}_{\text {ext }}$ the external radius of curvature of the guide. The following relations can thus be found (Fig. 2a):

$$
\rho=\sqrt{\mathrm{x}^{2}+\mathrm{y}^{2}}=\mathrm{R}_{\mathrm{ext}} \ln \left(\frac{\mathrm{z}}{\mathrm{R}_{\mathrm{ext}}}\right) \quad \text { and } \quad \theta=\operatorname{Arctan}\left(\frac{\mathrm{y}}{\mathrm{x}}\right)=\frac{\mathrm{v}}{\mathrm{R}_{\mathrm{ext}}}
$$

In the $\mathrm{z}$-space, the wave equation governing the propagation of light in the waveguide is $\left[\Delta_{\mathrm{x}, \mathrm{y}}+\right.$ $\left.\mathrm{k}_{0}^{2} \mathrm{n}^{2}(\mathrm{x}, \mathrm{y})\right] . \phi(\mathrm{x}, \mathrm{y})=0$, with $\phi \equiv \mathrm{E}$ or $\mathrm{H}$ that represent respectively the electric or magnetic field associated to the wave. By carrying out basic calculations based on partial differentials as $\frac{\partial}{\partial\left(\begin{array}{l}x \\ y\end{array}\right)}=$ $\frac{\partial u}{\partial\left(\begin{array}{l}x \\ y\end{array}\right)} \frac{\partial}{\partial u}+\frac{\partial v}{\partial\left(\begin{array}{l}x \\ y\end{array}\right)} \frac{\partial}{\partial v}$ plus the second order, it will occur $\frac{\partial^{2} u}{\partial\left(\begin{array}{l}x \\ y\end{array}\right)^{2}}=\frac{R_{\operatorname{ext}}\left(x^{2}+y^{2}\right)-2 R_{e x t}\left(\begin{array}{l}x \\ y\end{array}\right)^{2}}{\left(x^{2}+y^{2}\right)^{2}}$ and $\frac{\partial^{2} v}{\partial\left(\begin{array}{l}x \\ y\end{array}\right)^{2}}=$ $( \pm) \frac{2 x y R_{e x t}}{\left(x^{2}+y^{2}\right)^{2}}$. Then, a new Laplacian can be expressed by such a way with $\Delta_{x, y}=\Delta_{u, v} \exp (-2 u$ / $\left.\mathrm{R}_{\mathrm{ext}}\right)$. So the wave equation becomes:

$$
\left[\Delta_{\mathrm{u}, \mathrm{v}}+\mathrm{k}_{0}^{2} \cdot \mathrm{n}^{2}(\mathrm{u}) \exp \left(2 \mathrm{u} / \mathrm{R}_{\mathrm{ext}}\right)\right] \cdot \phi(\mathrm{u}, \mathrm{v})=0
$$

This equation is similar to the previous one but with a correction term on the index that becomes $n(u) \cdot \exp \left(u / R_{\text {ext }}\right)$. Thanks to the $C T$, it is possible to compute the modes in a curved waveguide by doing the calculation on a straight waveguide fitted with a modified index profile. This new profile will be sliced into virtual layers in order to carry out the multilayer matrix formalism. To each virtual layer $i$ with its index $n_{i}$, this formalism associates a matrix $M_{i}$ that can take both different forms according to the oscillating or evanescent natures of the fields [1]:

$$
M_{i}=\left(\begin{array}{cc}
\cos \left(q_{i} l_{i}\right) & \frac{j}{q_{i}} \sin \left(q_{i} l_{i}\right) \\
j q_{i} \sin \left(q_{i} l_{i}\right) & \cos \left(q_{i} l_{i}\right)
\end{array}\right), \text { for } n_{i}>n_{e f f}
$$

and,

$$
M_{i}=\left(\begin{array}{cc}
\cosh \left(\vartheta_{i} l_{i}\right) & \frac{j}{\vartheta_{i}} \sinh \left(\vartheta_{i} l_{i}\right) \\
j \vartheta_{i} \sinh \left(\vartheta_{i} l_{i}\right) & \cosh \left(\vartheta_{i} l_{i}\right)
\end{array}\right), \text { for } n_{i}<n_{\text {eff }}
$$

with, $j^{2}=-1$ the imaginary number, $q_{i}=\sqrt{k_{0}^{2} n_{i}^{2}-\beta^{2}}, \vartheta_{i}=j q_{i}=\sqrt{\beta^{2}-k_{0}^{2} n_{i}^{2}}, l_{i}$ the length of the layer, $n_{i}$ the index of the $i^{\text {th }}$ layer, $k_{0}=2 \pi / \lambda_{0}$ the wave-vector, $\lambda_{0}$ the wavelength, and $\beta=k_{0} n_{\text {eff }}$ the effective propagation constant that represent the eigenvalue of the system exactly as the effective 
index $\mathrm{n}_{\text {eff. }}$ The matrix describing the whole structure is the product of each one of the local matrices: $\bar{M}=\prod_{i=1}^{N} M_{i}=\left(\begin{array}{ll}m_{11} & m_{12} \\ m_{21} & m_{22}\end{array}\right)$ with $N$ the total number of layers (Fig. 2b).

One may consider now the eigenvalue equations of such a structure given by (TE/TM modes):

$$
\left\{\begin{array} { l } 
{ \mathrm { m } _ { 1 1 } - j \mathrm { jm } _ { 1 2 } + \frac { \mathrm { j } } { \mathrm { p } } \mathrm { m } _ { 2 1 } + \frac { \mathrm { r } } { \mathrm { p } } \mathrm { m } _ { 2 2 } = 0 } \\
{ \frac { \mathrm { p } } { \mathrm { n } _ { \mathrm { p } } ^ { 2 } } \mathrm { m } _ { 1 1 } - \mathrm { j } \frac { \mathrm { r } } { \mathrm { n } _ { \mathrm { r } } ^ { 2 } } \frac { \mathrm { p } } { \mathrm { n } _ { \mathrm { p } } ^ { 2 } } \mathrm { m } _ { 1 2 } - j \mathrm { m } _ { 2 1 } + \frac { \mathrm { r } } { \mathrm { n } _ { \mathrm { r } } ^ { 2 } } \mathrm { m } _ { 2 2 } = 0 }
\end{array} \text { and } \left\{\begin{array}{l}
\mathrm{p}=\sqrt{\beta^{2}-\mathrm{k}_{0}^{2} \mathrm{n}_{\mathrm{p}}^{2}} \\
\mathrm{r}=\sqrt{\beta^{2}-\mathrm{k}_{0}^{2} \mathrm{n}_{\mathrm{r}}^{2}}
\end{array}\right.\right.
$$

with, $n_{p}=n(-2 u)$ and $n_{r}=n(u=0)=n_{\text {clad }}$ into the TC index profile ( $4^{\text {th }}$ diagram in Fig. $\left.2 a\right)$.

Considering the previous equation (5) theory of multilayer structures, both terms $n_{p}$ and $n_{r}$ are respectively the indices of the substrate and superstrate. In the case of virtual layers on an index profile modified by a CT, as the index continuously varies, there is no such thing as constant substrate or superstrate, so the choice of the values of $n_{p}$ and $n_{r}$ has an influence on the calculation of eigenvalues per definition (see $n_{p}$ onto the $4^{\text {th }}$ diagram Fig. 2a). In this computing method, the chosen values are the lowest of each side of the cladding (that is $\mathrm{n}_{\mathrm{p}}$ on the left side area and $\mathrm{n}_{\mathrm{r}}=\mathrm{n}_{\text {clad }}$ on the right side area): then it depends on the lower bound chosen for the coordinate $-2 \mathrm{u}$ that has been taken equal to $-2 R_{\text {ext }} \ln \left(R_{\text {ext }} / R_{\text {int }}\right)$ so that each region (the left side, the core, and the right side) has the same length and the same number of i-iterations concerning the MMF. As an example, the computation of the TE mode eigenvalue equation for different values of $\mathrm{R}_{\text {ext }}$, $\mathrm{L}$ the width of the guide, and $\left(\mathrm{n}_{\text {core }} ; \mathrm{n}_{\text {cladd }}\right)$ yields relevant results summarized in Table 1 plus the comparison with COMSOL-3D computing.

It is clear that the results provided by the hybrid numerical method (HNM) present the same global kind of variation in function of the different opto-geometric parameters $\left(\mathrm{R}, \mathrm{L}, \mathrm{n}_{\mathrm{i}}, \lambda_{0}\right)$ of the structure, providing then a most valuable approximation of actual values. For the majority of the cases (see Table 1), precision can be observed weaker for $\mathrm{L}=2 \mu \mathrm{m}$ than for $\mathrm{L}=1 \mu \mathrm{m}$. Indeed, as the three regions (cladding on the left, core, and cladding on the right) feature a same length $\left(4^{\text {th }}\right.$ diagram, Fig. 2a), the value of the lower bound $n_{p}=n(-2 u)$ is defined by the effect of the CT and then the value of $R_{\text {ext }}$. The value $n_{p}$ (and also $\left(n_{r}-n_{p}\right)$ due to the fixed value of $n_{r}=n_{\text {clad }}=n(u=0)$ after the $C T$ ) for respectively a width $L=2 \mu \mathrm{m}$ will be below a value of $n_{p}$ for $L=1 \mu \mathrm{m}$. It is also remarkable that the greater $R_{\text {ext }}$ is, the more precise the results are. This can be explained by the fact that, the smaller $R_{\text {ext }}$ is, the more enforced will be the $\mathrm{CT}$ effect on the virtual index directly proportional to the factor $\mathrm{e}^{\left(\mathrm{u} / \mathrm{R}_{\text {ext }}\right)}$, which will increase the value $\left(\mathrm{n}_{\mathrm{r}}-\mathrm{n}_{\mathrm{p}}\right)=\left(\mathrm{n}_{\text {clad }}-\mathrm{n}(-2 \mathrm{u})\right)$. Indeed, if we consider the specific asymptotic case $R_{e x t} \rightarrow+\infty$, it will lead to $e^{\left(u / R_{e x t}\right)} \rightarrow 1$ and $n_{p}=n_{\text {clad }}=n_{r}$ representing no effect of CT. We can notice that the value $n_{p}$, defined by the effect of CT and also the distance $L$ according to Fig. 2a, is squared in the impact calculation of p into (5).

Concerning now the right area, as depicted on Fig. 2a, the index is increased on such area of the waveguide in the w-plane, which corresponds to the external side of the guide in the z-space, so we can expect the light to be deported outwards, in the same way that one could figure about centrifugal acceleration (Fig. 3). The mechanism to shape successively the amplitude of the discretized optical mode by classical MMF is defined by the procedure: Field $_{\text {initial }=1}=\overline{\mathrm{M}}$. Field final $=\mathrm{N}_{\text {}}$. The values of $\Delta \mathrm{x}_{\text {peak }}$ effectively highlight such a phenomenon obviously, and, as may be expected, the greater $\mathrm{R}_{\text {ext }}$ is considered, the smaller $\Delta \mathrm{x}_{\text {peak }}$ is, varying down to zero as $\mathrm{R}_{\mathrm{ext}}$ tends to infinity, which yields the previous asymptotic case of a straight waveguide. As regards index contrast, we can also point out the fact that for $\left(\mathrm{n}_{\text {core }} ; \mathrm{n}_{\text {cladd }}\right)=(2 ; 1.5), \Delta \mathrm{x}_{\text {peak }}$ is greater than for $\left(\mathrm{n}_{\text {core }} ; \mathrm{n}_{\text {cladd }}\right)=(2 ; 1)$ or $\left(\mathrm{n}_{\text {core }} ; \mathrm{n}_{\text {cladd }}\right)=(2.5 ; 1.5)$. Indeed, as the difference between $\mathrm{n}_{\text {core }}$ and $\mathrm{n}_{\text {cladd }}$ is reduced, the mode becomes less guided, so the more it is deported outwards. To conclude, all the previous classical 
physical behaviors in propagation are fortunately found in the results of such hybrid numerical method $\mathrm{HNM}=(\mathrm{MMF} o \mathrm{CT})$ computing without requiring a tremendous power of calculation. This hybrid method allows valuable approximation of the characteristics of such structures.

\section{References}

[1] M.J. Adams, Introduction to optical waveguides, John Wiley \& Sons, 1981.

[2] T. Tamir, Guided-wave optoelectronics, Springer-Verlag $2^{\mathrm{d}}$ Ed, 1990.

[3] A.W. Snyder, J.D. Love, Optical waveguide theory, Kluwer Academic Publishers $2^{\mathrm{d}}$ Ed, 2000.

[4] B. Bêche, E. Gaviot, A. Renault, J. Zyss, F. Artzner, Another way to shape the comprehensive analytical approach describing electromagnetic energy distribution through four-slab-layer structures, Optik 121 (2010) 188-194.

[5] T. Begou, B. Bêche, N. Grossard, J. Zyss, A. Goullet, G. Jézéquel, E. Gaviot, Marcatili’s extended approach: comparison to semi-vectorial methods applied to pedestal waveguide design, J. Opt. A: Pure Appl. Opt. 10 (2008) 055310.1-055310.10.

[6] Murray R. Spiegel, Variables complexes, Schaum -Mac Graw-Hill New-York, 1973.

[7] P. Yeh, A. Yariv, C-S. Hong, Electromagnetic propagation in periodic stratified media. I. General theory, Opt Soc. Am., 67 (1977) 423-438.

[8] www.comsol.com 


\section{Figure caption:}

Figure 1: Schematic representation of an infinite slab curved waveguide.

Figure 2: Explanations about both elements of the hybrid method. a) conformal transformation (CT) of the Z-plane to the W-plane. The Z-plane corresponds to the real configuration where the waveguide is curved, on which the operation $w=R_{\text {ext }} \cdot \ln \left(z / R_{e x t}\right)$ is applied prior to further calculations on a waveguide in the $\mathrm{W}$-plane that is devoid of any radius of curvature, but is fitted with a modified index profile. b) Multilayer matrix formalism (MMF) that allow to slice the index profile into virtual layers so as to determine the eigenvalues (effective propagation constant or effective index) and the eigenvectors (optical field) in such a structure.

Figure 3: Representation of the optical field in a non-curved waveguide (dashed line) and in a waveguide with a $10 \mu \mathrm{m}$ radius of curvature (continuous line). The term $\Delta \mathrm{x}_{\text {peak }}$ represents the shift of position of the maximum regarding intensity between the two cases. 
Fig 1:

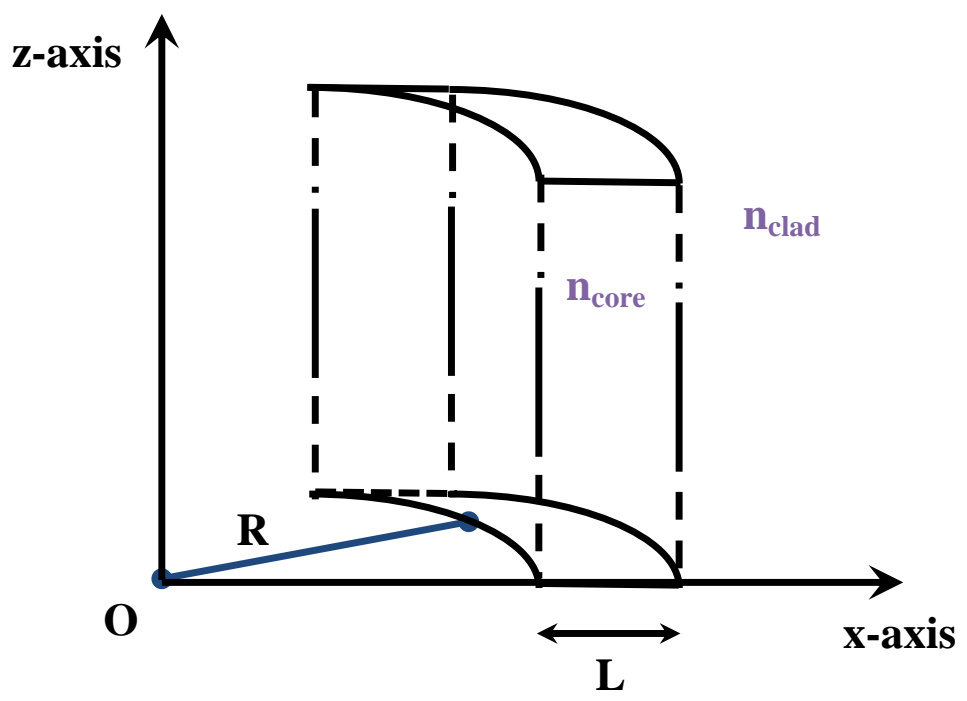


Fig 2:

a)

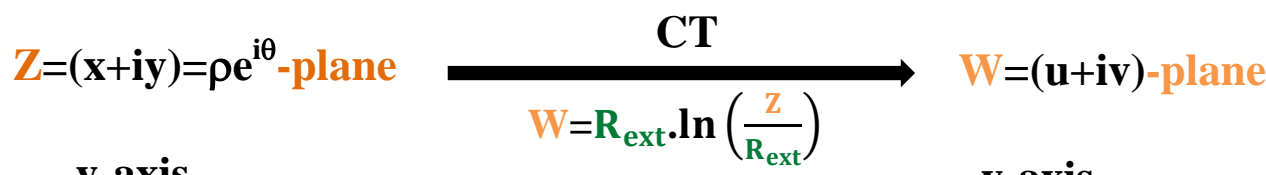
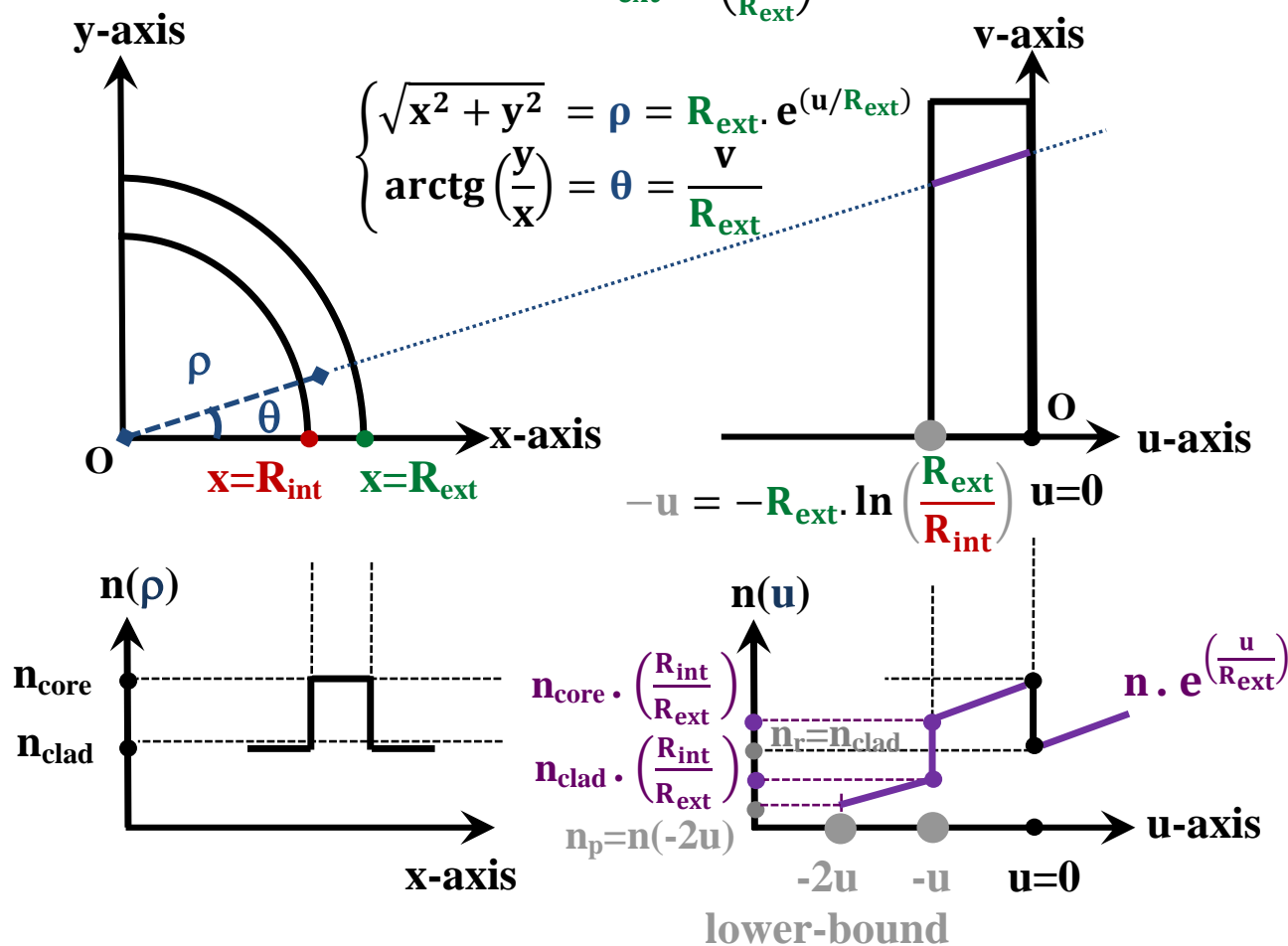

b)

Multilayer formalism applied to CT and virtual n(u)

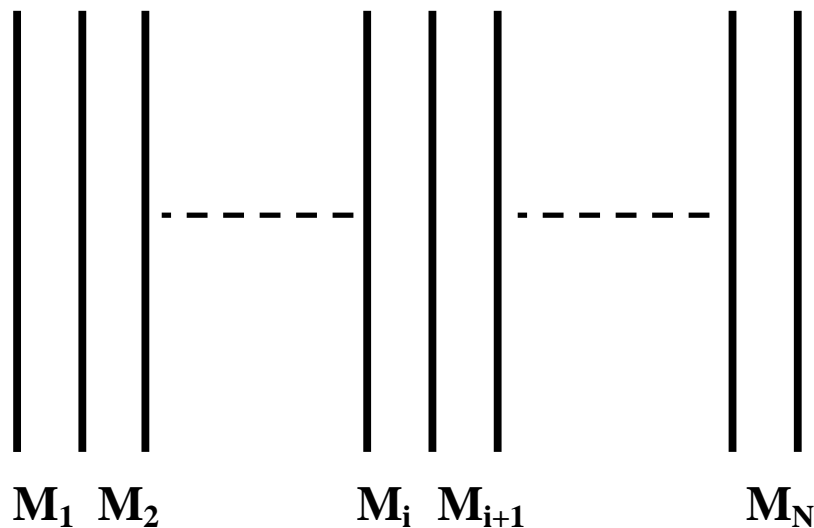


Fig 3:

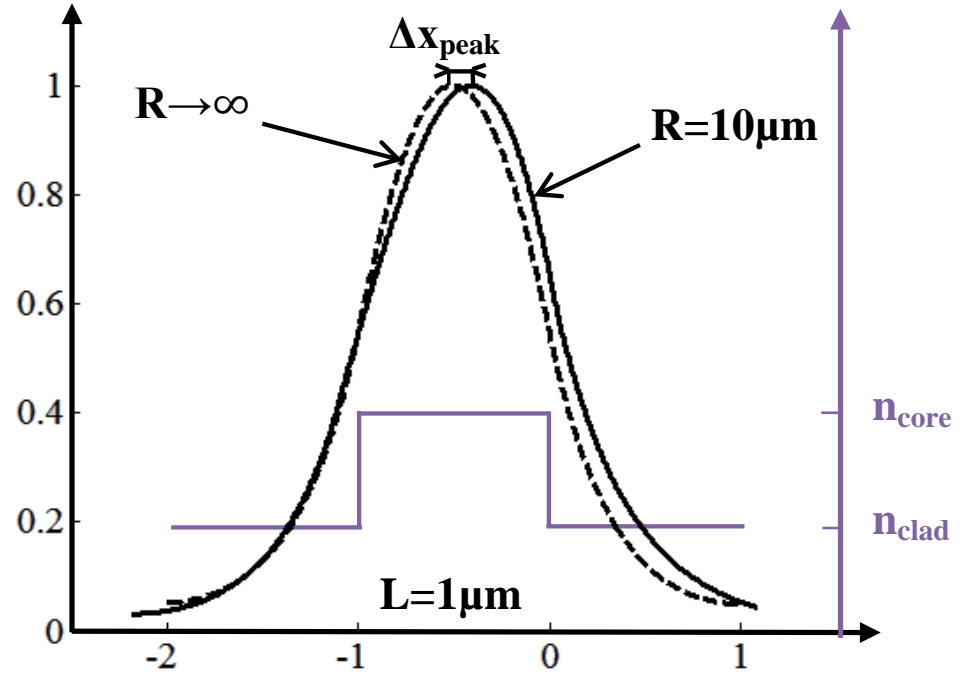


Table caption:

As an example, this table represents the eigenvalues of the $\mathrm{TE}_{0}$-modes for different width $(\mathrm{L})$ of the guide and for different indices regarding the cladding $\left(\mathrm{n}_{\text {clad }}\right)$ and the core $\left(\mathrm{n}_{\text {core }}\right)$ for an optical excitation of wavelength $\lambda_{0}=1 \mu \mathrm{m}$. These values are compared to those given by COMSOL-3D software. The $\Delta \mathrm{x}_{\text {peak }}$ represents the shift between the positions of the maxima of intensity of the light and the center of the guide (where the maximum is when considering a straight guide). 
Table:

\begin{tabular}{|c|c|c|c|c|c|c|}
\hline$\underset{(\mu \mathrm{m})}{\mathbf{R}}$ & $\underset{(\mu \mathrm{m})}{\mathbf{L}}$ & $\left(\mathbf{n}_{\text {core }} ; \mathbf{n}_{\text {clad }}\right)$ & $\begin{array}{l}\mathbf{n}_{\text {eff-TE0 }} \\
(H N M)\end{array}$ & $\begin{array}{c}\mathbf{n}_{\text {eff-TE0 }} \\
(C O M S O L)\end{array}$ & $\left(\frac{H N M-C O M S O L}{C O M S O L}\right) \%$ & $\begin{array}{c}\Delta \mathbf{x}_{\text {peak }} \\
(\boldsymbol{\mu m}) \\
(H N M)\end{array}$ \\
\hline \multirow{6}{*}{10} & \multirow{3}{*}{1} & $(2 ; 1)$ & 1.6844 & 1.9529 & 13.75 & 0.074 \\
\hline & & $(2.5 ; 1.5)$ & 2.1925 & 2.0021 & 9.51 & 0.095 \\
\hline & & $(2 ; 1.5)$ & 1.7444 & 1.9571 & 10.87 & 0.142 \\
\hline & \multirow{3}{*}{2} & $(2 ; 1)$ & 1.7721 & 1.9850 & 10.73 & 0.439 \\
\hline & & $(2.5 ; 1.5)$ & 2.2581 & 1.9938 & 13.26 & 0.527 \\
\hline & & $(2 ; 1.5)$ & 1.7930 & 1.9856 & 9.70 & 0.549 \\
\hline \multirow{6}{*}{15} & \multirow{3}{*}{1} & $(2 ; 1)$ & 1.7079 & 1.9540 & 12.54 & 0.052 \\
\hline & & $(2.5 ; 1.5)$ & 2.2223 & 2.0000 & 11.11 & 0.067 \\
\hline & & $(2 ; 1.5)$ & 1.7642 & 1.9582 & 9.91 & 0.093 \\
\hline & \multirow{3}{*}{2} & $(2 ; 1)$ & 1.8095 & 1.9860 & 8.89 & 0.320 \\
\hline & & $(2.5 ; 1.5)$ & 2.3008 & 1.9977 & 15.17 & 0.406 \\
\hline & & $(2 ; 1.5)$ & 1.8253 & 1.9866 & 8.12 & 0.416 \\
\hline \multirow{6}{*}{$\infty$} & \multirow{3}{*}{1} & $(2 ; 1)$ & 1.7618 & 1.8106 & 2.70 & 0.000 \\
\hline & & $(2.5 ; 1.5)$ & 2.2914 & 2.3477 & 2.40 & 0.000 \\
\hline & & $(2 ; 1.5)$ & 1.8160 & 1.8354 & 1.06 & 0.000 \\
\hline & \multirow{3}{*}{2} & $(2 ; 1)$ & 1.9124 & 1.9467 & 1.76 & 0.000 \\
\hline & & $(2.5 ; 1.5)$ & 2.4260 & 2.4565 & 1.24 & 0.000 \\
\hline & & $(2 ; 1.5)$ & 1.9239 & 1.9498 & 1.33 & 0.000 \\
\hline
\end{tabular}

